\title{
Endovascular arch replacement with a dual branched endoprosthesis
}

\author{
Ciro Ferrer ${ }^{1}$, Piergiorgio Cao $^{2}$ \\ ${ }^{1}$ Unit of Vascular Surgery, Department of Surgery "Pietro Valdoni”, Sapienza University of Rome, Rome, Italy; ${ }^{2}$ Mater Dei Hospital, Rome, Italy \\ Correspondence to: Ciro Ferrer. Unit of Vascular Surgery, Department of Surgery "Pietro Valdoni”, Sapienza University of Rome, Italy. \\ Email: cfrrr83@gmail.com.
}

Background: In the light of current evidence, endovascular repair of aortic arch pathologies with custommade devices should be considered a valid alternative to decrease operative mortality and morbidity associated with open or hybrid repair. Currently, two double inner branch devices are available on the market. Some papers from multicenter experiences have been published about the use of Cook device. We report our singlecenter experience with Bolton double branch stent graft in the treatment of aortic arch disease.

Methods: Between 2013 and 2016, nine high-risk patients with arch pathology were treated in our center with a Bolton custom-made branched device. Among these, two with a single branch model were excluded, leaving a subgroup of seven patients of this study.

Results: Out of the seven male patients (mean age, 76; range, 70-85) included in the study, two (28\%) died perioperatively after stroke (14\%) and retrograde dissection (14\%), respectively. No other death, major complications, including aneurysmal diameter evolution and branch related complications, or secondary intervention was recorded at a mean follow up of 24 [6-53] months.

Conclusions: Despite the small sample size, our results are in line with the early experiences published on this technique. Endovascular repair of aortic arch disease with custom-made branched devices should always be considered to give high-risk patients a chance of repair.

Keywords: Aortic arch; aortic aneurysm; endovascular repair; branched stent graft; custom-made stent graft

Submitted Jan 26, 2018. Accepted for publication Apr 09, 2018.

doi: 10.21037/acs.2018.04.08

View this article at: http://dx.doi.org/10.21037/acs.2018.04.08

\section{Introduction}

Any treatment of aortic arch disease is demanding and technically challenging. Complete replacement of the aortic arch is one of the most complex operations in cardiovascular surgery and remains probably the last hurdle in providing patients with an endovascular option for the entire aorta. Despite recent formidable improvements in surgical procedures and the development of hybrid techniques, perioperative mortality and major morbidity remain significant (1-3). Endovascular strategies are showing promise in treating the aortic arch with similar or even lower mortality and morbidity compared to surgical repair, despite addressing a more complex patient population. This article reports clinical results and discusses technical issues of endovascular arch repair with a custom-made double inner branch device in a single-center experience.

\section{Methods}

All patients with aortic arch disease who were treated with the Bolton double branch device (Bolton Medical España, Barcelona, Spain; Bolton Medical, Inc., Sunrise, FL, USA) in a single center, between 2013 and 2016, were included in this study. Each patient was assessed according to a multidisciplinary approach and deemed unfit for open surgery because of comorbidities or previous sternotomy. Preoperative evaluation of computed tomographic angiography (CTA) was performed for all patients with Aquarius iNtuition workstation (Terarecon, Inc., Foster 


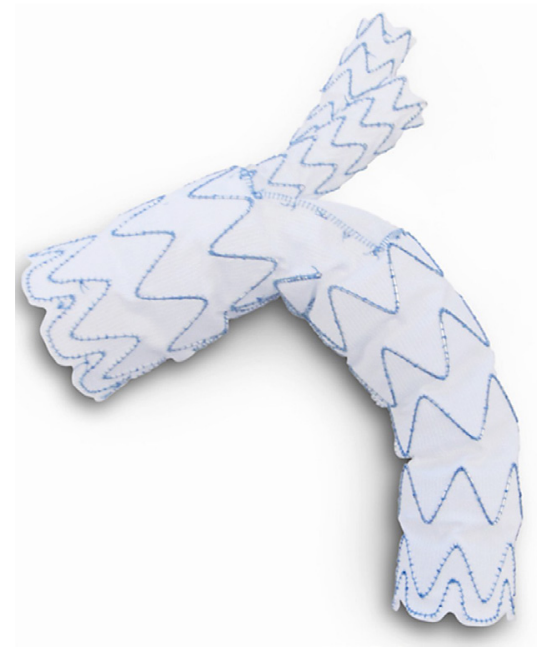

Figure 1 Bolton double branch device.

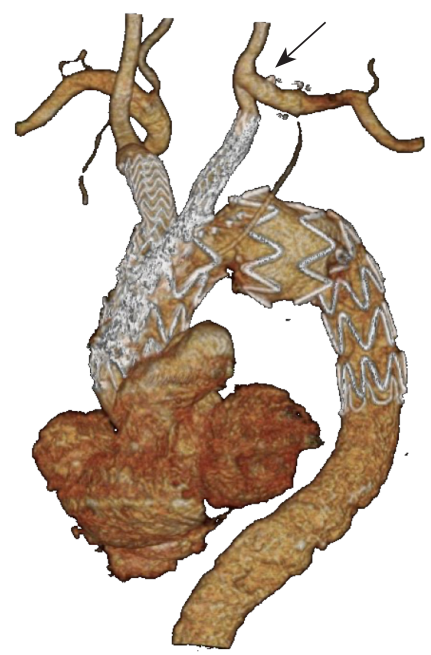

Figure 2 3D reconstruction of post-operative CTA after double branch arch procedure with Bolton device. LCCA-LSA bypass (arrow). CTA, computed tomographic angiography; LCCA, left common carotid artery; LSA, left subclavian artery.

city, CA, USA), and a center-lumen line tool was used to draw the endograft scheme. Informed consent was obtained for each patient. Data were prospectively collected in an electronic database and retrospectively reviewed.

\section{Device}

The Bolton double branch arch device is a custom-made thoracic stent graft based on the Relay non bare stent
(NBS) platform (Bolton Medical España, Barcelona, Spain; Bolton Medical, Inc., Sunrise, FL, USA), and therefore features the same specific characteristics such as a dual sheath, a pre-curved inner nitinol catheter, and a proximal capture system (Figure 1). The distinguishing feature is a wide window in the superior aspect of the endograft that can accept the two inner channels. The window and the proximal end of each internal branch, as well as the ends of the endograft, are equipped with radiopaque markers that make them visible under fluoroscopy. Both tunnels are equipped with barbs in order to prevent potential disconnection of the branches.

\section{Procedure}

The use of a double branch arch stent graft requires a prior left subclavian artery (LSA) revascularization performed by bypass or transposition (Figure 2). The endovascular stage is performed under general anesthesia and continuous cerebral monitoring with INVOS Cerebral Oximetry System (Medtronic, Inc. Minneapolis, MN, USA). The aortic main body and the bridging stents are usually flushed before insertion with carbon dioxide followed by saline solution. One femoral artery is approached by surgical exposure and used to insert the main body over a stiff guide-wire positioned through the aortic valve into the left ventricle. The contralateral femoral artery is percutaneously approached with a 5-F sheath for an angiographic check. The right axillary artery and the left brachial artery (or alternatively both common carotid arteries) are used as access vessels to catheterize the inner channels. With an activated clotting time test $\geq 300$ seconds, two guide-wires are placed in the ascending aorta from the upper accesses to mark the origin of the innominate artery (IA) and left common carotid artery (LCCA). A pigtail catheter is placed into the apex of the left ventricle from the femoral access, and a stiff guide-wire (Lunderquist; Cook Medical, Bloomington, IN, USA) is advanced through this catheter. Under fluoroscopy, the orientation of the branched stent graft is verified outside the patient and then delivered over the stiff guide-wire to the aortic arch. The tapered short tip is brought through the aortic valve into the left ventricle. The correct projection to best display the origin of coronary ostium and IA is now to be set. An angiogram is performed, the markers on the branches are adequately positioned, and the stent graft is deployed under rapid pacing or other cardiac output suppression technique. Normal cardiac output is resumed while the tip of the delivery system and 
the stiff guide-wire are retrieved from the left ventricle. The inner branches are catheterized from the target vessels. Two sheaths are positioned into the channels. Appropriate custom-made or off-the-shelf bridging limbs are used for IA stenting, while covered stents are usually preferred for LCCA. Before deployment of the bridging stents, it is necessary to test the correct engagement of the channel and

\begin{tabular}{|ll}
\hline \multicolumn{2}{|l}{ Table 1 Demographics and baseline characteristics of the sample } \\
\hline Characteristics & Values \\
\hline $\mathrm{N}$ & 7 \\
\hline Male & $7(100 \%)$ \\
\hline Mean age & $76[70-85]$ \\
\hline Smoking & $2(28 \%)$ \\
\hline Hypertension & $7(100 \%)$ \\
\hline Coronary disease & $3(42 \%)$ \\
\hline Prior coronary bypass & $2(28 \%)$ \\
\hline Chronic obstructive pulmonary disease & $3(42 \%)$ \\
\hline Dyslipidemia & $2(28 \%)$ \\
\hline Diabetes & $0(0 \%)$ \\
\hline Renal insufficiency & $0(0 \%)$ \\
\hline Prior cerebral event & $0(0 \%)$ \\
\hline Prior aortic surgery & $1(14 \%)$ \\
\hline Prior ascending aortic surgery & $0(0 \%)$ \\
\hline Aneurysm & $7(100 \%)$ \\
\hline Chronic dissection & $0(0 \%)$ \\
\hline
\end{tabular}

ensure that the proximal end of the stent is within 1-2 mm of the proximal gold markers in the respective tunnel and that there is adequate overlap with the target vessel. Balloon angioplasty is performed at overlapping zones between the aortic main body and the bridging stents and at the distal landing in the target vessels. Dilatation of the proximal end of the aortic main body, using an aortic balloon, is not recommended.

\section{Results}

Out of the 13 patients treated with a double branch arch device during the study period, seven received a Bolton double branch endograft and represent the subject of this study. Two other patients treated with a Bolton single branch device were excluded from the analysis. The mean age of the seven male patients was 76 [70-85] years. Baseline comorbid status and anatomical characteristics of the sample are reported in Tables 1 and 2 , respectively. One patient was previously operated for a type III thoracoabdominal aneurysm by open surgery. The indication for treatment was atherosclerotic aneurysm in all cases. No patient was treated for aortic dissection and none had had a previous ascending aorta operation. All patients received a LCCA-LSA bypass as first step. Endovascular step was carried out after a mean interval of 7 [3-21] days. The LSA stump was always occluded by a vascular plug. In all cases, the aortic main body was inserted via the common femoral artery, and the cardiac output reduction was achieved by rapid pacing. The right axillary artery was used to access the IA tunnel in all cases, while the LCCA tunnel was catheterized from the left brachial

\begin{tabular}{|c|c|c|c|c|c|c|}
\hline Patient & $\begin{array}{l}\text { Ascending aorta } \\
\text { diameter }(\mathrm{mm})\end{array}$ & $\begin{array}{l}\text { IA diameter } \\
(\mathrm{mm})\end{array}$ & $\begin{array}{l}\text { LCCA diameter } \\
(\mathrm{mm})\end{array}$ & $\begin{array}{l}\text { Aortic main } \\
\text { body size }(\mathrm{mm})\end{array}$ & $\begin{array}{l}\text { IA stent } \\
\text { graft size }(\mathrm{mm})\end{array}$ & $\begin{array}{l}\text { LCCA stent } \\
\text { graft size }(\mathrm{mm})\end{array}$ \\
\hline$\# 1$ & 30 & 18 & 10 & $36-40 \times 260$ & 20 & 10 \\
\hline \#2 & 35 & 16 & 10 & $42-32 \times 240$ & 18 & 10 \\
\hline \#3 & 43 & 16 & 12 & $46-36 \times 250$ & 18 & 13 \\
\hline$\# 4$ & 38 & 18 & 10 & $42-36 \times 260$ & 20 & 12 \\
\hline$\# 5$ & 34 & 20 & 12 & $40-36 \times 220$ & 23 & 12 \\
\hline$\# 6$ & 39 & 17 & 7 & $44-36 \times 270$ & 18 & 10 \\
\hline \#7 & 34 & 12 & 7 & $38-26 \times 255$ & 20 & $9+12$ \\
\hline
\end{tabular}

IA, innominate artery; LCCA, left common carotid artery. 


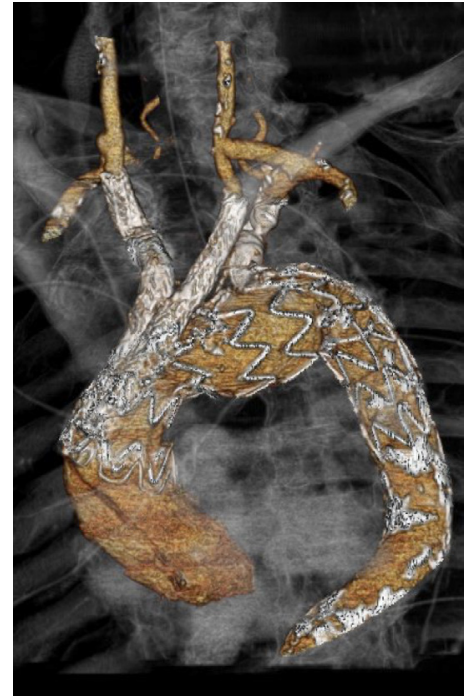

Figure 3 Ascending aorta retrograde dissection (arrow) detected at early post-operative CTA after double branch implantation (Bolton device). CTA, computed tomographic angiography.

artery (through the bypass) in five of the seven cases and from the cervical portion of the LCCA in the remaining two. The IA was bridged with a custom-made Bolton limb in three cases and a Gore Excluder limb (W.L. Gore \& Associates, Flagstaff, AZ, USA) in four cases. Meanwhile, the LCCA was stented by a Fluency stent graft (Bard Peripheral Vascular, Tempe, AZ, USA) in three cases. a Viabahn stent graft (W.L. Gore \& Associates, Flagstaff, AZ, USA) in three cases, and a combination of Viabahn and BeGraft (Bentley InnoMed, Hechingen, Germany) in one case. All the Viabahn stent grafts were reinforced with a bare metal stent. The mean fluoroscopy time was 46 [39-55] minutes, and the mean amount of contrast medium was 178 [120-210] mL.

Technical success, intended as correct deployment of aortic and supra-aortic stent grafts, exclusion of the aneurysm and patency of supra-aortic vessels, was achieved in $100 \%$ of cases. Two of the seven patients $(28 \%)$ died perioperatively. The first death was due to a respiratory arrest 72 hours after the procedure in a patient diagnosed with an ischemic lesion of the basal ganglia at magnetic resonance imaging (MRI). The other death occurred for aortic rupture at 48 hours in a patient just diagnosed with ascending aorta retrograde dissection at CTA (Figure 3). No other neurologic events, nor other retrograde dissections, occurred perioperatively. Moreover, there were no cases of cardiac, renal or access related complications, and no spinal cord ischemia. The mean postoperative length of stay for the five survivors was seven [4-14] days, including a mean intensive care unit stay of 1.5 [1-3] days. No early reintervention was performed. All the 7 patients underwent a postoperative CTA before discharge. Except for the patient who presented with a retrograde dissection, no branch vessel related complication, endoleak, or other vascular complications were observed at CTA.

Mean follow up time was 24 [6-53] months. One patient was lost to follow up. No patient died among the remaining four and none experienced any neurologic events or required secondary interventions. No aneurysmal diameter evolution or branch related complication was observed at imaging follow up.

\section{Discussion}

Although open repair remains the gold standard of care for patients with limited comorbidities, alternative options have been proposed to address more complex patient populations. Several endovascular repair strategies have emerged in the treatment of aortic arch disease, including hybrid repair, chimney techniques, and use of custommade devices. Endovascular strategies offer advantages over open repair, as they are minimally invasive and do not require hypothermic circulatory arrest or rerouting of aortic blood flow. Several of these techniques have proven technically successful with promising early and midterm results, whereas others have been associated with longterm durability concerns. The endovascular approach to arch pathology with custom-made inner branch devices is currently reserved for patients deemed unfit for open or hybrid repair. The major concern in complex arch stent grafting derives from the proximity of the aortic valve and coronary ostia, and the potential neurologic complications inflicted by a hypothetical flow-limiting stent graft misalignment or embolization event. Moreover, given the catastrophic consequences potentially associated with loss of device seal or device migration in the aortic arch, patient selection according to the availability of a healthy seal zone ( $\leq 38 \mathrm{~mm}$ in diameter) of at least $25 \mathrm{~mm}$ in length is crucial for immediate technical success and long-term durability. In addition, despite significant efforts to minimize the profile of the device by using low-profile graft fabric and stents, the sheath size for an arch branched device remains as large as 22-24 F (inner diameter). Practicability of access routes remains another reason for concern, as these large-profile systems have typically been associated with increased access 
site complications due to an increased need for vascular cutdowns and conduits.

Apart from some few case reports about the Bolton arch branch device, the majority of published papers about this technique regards the Cook platform. The first clinical use of this device was reported by Lioupis et al. in 2012 (4). In this initial case series of six patients, 11 of the 12 branches $(91.6 \%)$ were successfully cannulated and stented. In one case, the IA branch could not be catheterized, and the patient needed a femoral-axillary bypass. The same patient developed a stroke. Other major complications noted in this series included a right cerebellar stroke, and a type I endoleak in a patient with an ascending aorta as large as $39 \mathrm{~mm}$. Although a proximal seal could most certainly be initially obtained, the long-term durability in such a large-caliber aorta is unknown. In our series, two patients presented a native ascending aorta larger than $38 \mathrm{~mm}$ of which one larger than $40 \mathrm{~mm}$ (Table 2). None of these reported a proximal endoleak, although a fatal retrograde dissection was recorded in a patient with $43 \mathrm{~mm}$ ascending aorta. Landing in such large ascending aortas will likely be associated with increased complications, as vessels of this size are typically indicative of disease. In our patient, a faulty non-perpendicular deployment of the first sealing stent was the most likely cause of intimal rupture in an already diseased aorta.

Haulon et al. reported a retrospective, multicenter experience of 38 high-risk patients treated with the Cook arch branched graft, including the aforementioned series of six patients (5). It is important to note that this case series is inclusive of the entire learning curve with this device. Technical success was achieved in 32 of 38 patients $(84.2 \%)$. Technical failures included three deaths within 24 hours of the procedure, one proximal type I endoleak, one failure to cannulate the IA branch, and one conversion to chimney technique. Five patients (13.2\%) died within 30 days of the procedure, and there were six cases with cerebrovascular complications noted in the follow-up. Although the difference was not statistically significant, mortality was $30 \%$ and $7.1 \%$ in the first 10 and last 28 patients treated, respectively. When combined together, the risk of early mortality or neurologic complications was statistically higher in the first 10 patients $(\mathrm{P}=0.019)$ and patients with ascending aorta diameters $>38 \mathrm{~mm}(\mathrm{P}=0.026)$. Among our patients, only one reported a cerebrovascular event, which led to death three days postoperatively, due to respiratory arrest caused by an ischemic lesion of the basal ganglia. This patient is part of our earliest experience, when device flushing with carbon dioxide was not routinely performed. Kölbel and co-authors reported in a group of 36 patients treated with ascending aorta or arch devices flushed with carbon dioxide, just one minor stroke in a patient with a highly calcified aortic arch (6). Of course, our sample is too small to reach statistical significance in determining the use of carbon dioxide as a protective factor in the prevention of air embolism-related neurologic events.

These initial reports, in line with the results of our study, demonstrated that with proper operator training, advanced technical refinement, and careful patient selection, aortic arch repair could be extended to patients who are not candidates for open surgical repair. The effect of learning curve was emphasized in a recent paper by Spear et al regarding the subsequent results for arch aneurysm repair with inner branched endografts (7). The authors reported in this study the results of their more recent experience, including 27 patients coming from three high-volume aortic centers. Although some authors of this study were the same of the previous paper, none of the 38 patients included in the aforementioned pioneering study were reported in this paper. Comparing these two samples, at 30 days, the rate of cerebrovascular events was comparable $(15.8 \% v s$. $11.1 \%, \mathrm{P}=0.6$ ), while there was a trend towards a decrease in endoleak rate $(28.9 \%$ vs. $11.1 \%, \mathrm{P}=0.08)$ in more recent group. In particular, no early mortality was observed in the late phase of their experience, compared with five cases $(13.2 \%)$ in the pioneering study $(\mathrm{P}=0.05)$. Overall mortality, during follow up, was significantly decreased in the late experience ( $3.7 \%$ vs. $23.6 \%, \mathrm{P}=0.02)$. Early secondary interventions, reported in the paper by Spear et al., were four $(14.8 \%)$. Of these, two sternotomies were performed to rule out a left ventricular false aneurysm. One of these two patients experienced a major stroke. Although, in our study, no early or late re-intervention was reported, one patient experienced an ascending aorta retrograde dissection. Unfortunately, that patient died just before undergoing surgical correction. This underlines the importance of a multidisciplinary team. Cardiothoracic surgeons should thus be involved in the whole process, from patient selection to post-operative care. Centers with no on-call cardiothoracic surgeon should not perform these repairs.

In our center, during the same study period, besides the seven patients included in the present report, another six were treated with the Cook arch branched device. Although there is no particular reason for the choice of endograft model, no patient in the group treated with Cook device presented an 


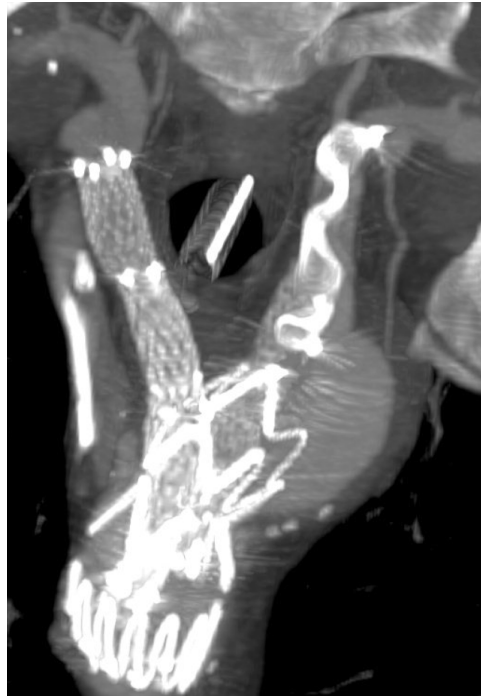

Figure 4 Partial coverage of the left vertebral artery by the vascular plug deployed in the LSA in a double branch procedure with Cook device. LSA, left subclavian artery.

ascending aorta larger than $38 \mathrm{~mm}$. In contrast to the Bolton group, the indication for treatment was an atherosclerotic aneurysm in five patients and residual type A chronic dissection in one. The dissected patient was the only one of this group to experience a major complication, which was a posterior stroke due to a partial occlusion of the left vertebral artery by the vascular plug deployed in the LSA (Figure 4). No patient died or had any other major complications in this subgroup at a mean follow up of 22 [19-38] months. Unfortunately, because of the small sample size, which is a limitation of the present study, no statistical analysis or direct comparison between these two groups was possible.

\section{Conclusions}

Despite the small sample size, our results are consistent with the early published experiences on this technique. Global trends confirm the effect of the learning curve and the importance of continuous technique refinement in determining improvements in major outcomes. Results from published series demonstrate that arch endografting is a feasible option and compares favorably with open and hybrid repairs for patients unfit for open surgery. In our series, technical success was always achieved when inclusion and exclusion criteria, as reported in larger series, are respected. Endovascular repair of aortic arch disease with inner branch devices should always be considered to give high-risk patients a chance of repair.

\section{Acknowledgements}

None.

\section{Footnote}

Conflicts of Interest: C Ferrer discloses proctoring and speaker's fees from Bolton Medical; P Cao discloses proctoring from Cook.

\section{References}

1. Iba Y, Minatoya K, Matsuda H, et al. Contemporary open aortic arch repair with selective cerebral perfusion in the era of endovascular aortic repair. J Thorac Cardiovasc Surg 2013;145:S72-7.

2. Benedetto U, Melina G, Angeloni E, et al. Current results of open total arch replacement versus hybrid thoracic endovascular aortic repair for aortic arch aneurysm: a meta-analysis of comparative studies. J Thorac Cardiovasc Surg 2013;145:305-6.

3. De Rango P, Cao P, Ferrer C, et al. Aortic arch debranching and thoracic endovascular repair. J Vasc Surg 2014;59:107-14.

4. Lioupis C, Corriveau MM, MacKenzie KS, et al. Treatment of aortic arch aneurysms with a modular transfemoral multibranched stent graft: initial experience. Eur J Vasc Endovasc Surg 2012;43:525-32.

5. Haulon S, Greenberg RK, Spear R, et al. Global experience with an inner branched arch endograft. J Thorac Cardiovasc Surg 2014;148:1709-16.

6. Kölbel T, Rohlffs F, Wipper S, et al. Carbon Dioxide Flushing Technique to Prevent Cerebral Arterial Air Embolism and Stroke During TEVAR. J Endovasc Ther 2016;23:393-5.

7. Spear R, Haulon S, Ohki T, et al. Editor's Choice Subsequent Results for Arch Aneurysm Repair with Inner Branched Endografts. Eur J Vasc Endovasc Surg 2016;51:380-5.

Cite this article as: Ferrer C, Cao P. Endovascular arch replacement with a dual branched endoprosthesis. Ann Cardiothorac Surg 2018;7(3):366-371. doi: 10.21037/ acs.2018.04.08 